\title{
Core competencies acquired in indigenous training of traditional health practitioners in Kwazulu-Natal
}

\author{
Mbatha Nompumelelo ${ }^{1}$, Exnevia Gomo ${ }^{2}$, Nceba Gqaleni ${ }^{3,4}$, Mlungisi Ngcobo ${ }^{3}$
}

1. University of KwaZulu-Natal College of Health Sciences, Traditional medicine Laboratory

2. School of Nursing \& Public Health, College of Health Sciences, University of KwaZulu-Natal, South Africa, Traditional Medicine Laboratory,

3. Traditional Medicine Laboratory, School of Nursing \& Public Health, College of Health Sciences, University of KwaZulu-Natal, South Africa.

4. Department of Public Management and Economics, Faculty of Management Sciences, Durban University of Technology, Durban, South Africa

\begin{abstract}
Introduction: Despite the recognition of Traditional Medicine systems as a critical component of health care by the WHO and the African Union, its integration into the health care mainstream remains very subdued in South Africa. This is partly due to the lack of empirical data pertinent to traditional healer training that could inform the accreditation process.

Objective: To determine core competencies acquired by Traditional Health Practitioners (THP) of KwaZulu-Natal Province, South Africa during their apprenticeship.

Materials and methods: Purposeful, convenient and snowballing sampling and the sequential data collection methods of questionnaires, journaling and focus groups was used to collect data from the THP tutors and their trainees in rural, peri-urban and urban areas of eThekwini and uThungulu Districts of Kwa Zulu Natal (KZN).

Results: Eleven core competencies were identified: consultation, diagnoses, holistic patient care and treatment, integrative and holistic healing, application of healing procedures and cultural rituals, spiritual development, ethical competencies, problem solving, herbalism, ancestral knowledge and end of life care.

Conclusion: The apprenticeship of THPs in KZN is based on eleven core competencies. These competencies are fundamental pillars for critical health care provided by THPs and are crucial for setting standards for the accreditation of traditional training in South Africa if the THP Act 22 of 2007 is to achieve its purpose of providing for the management of and control over the registration, training and conduct of the practitioners. Hence, the appointed interim THP Council should include the identified competencies when articulating bases for accreditation of the training and assessments.
\end{abstract}

Keywords: Indigenous training, traditional health practitioners, Kwazulu-Natal.

DOI: https://dx.doi.org/10.4314/ahs.v19i4.32

Cite as: Nompumelelo M, Gomo E, Gqaleni N, Ngcobo M. Core competencies acquired in indigenous training of traditional health practitioners in Kwazulu-Natal. Afri Health Sci.2019;19(4):3100-3106. https:/ / dx.doi.org/10.4314/ abs.v19i4.32

\section{Background}

Traditional Medicine (TM) has sustained the wellbeing of indigenous communities for centuries. It is estimated that at least $80 \%$ of the African population use TM. ${ }^{1}$ This

\section{Corresponding author:}

Mbatha Nompumelelo,

University of KwaZulu-Natal

College of Health Sciences,

Traditional medicine Laboratory

Email: mpumembatha1@gmail.com,

971122288@stu.ukzn.ac.za signifies the important role played by TM in the general health of the African population. The World Health Organisation (WHO) and the African Union (AU) and its member states recognise TM systems as a critical part of integrative health care., ${ }^{2,3}$ However, for several reasons, South Africa is still struggling with issues of integrating TM into the national health care mainstreams. These reasons include the recognition, registration and accreditation of THPs. Whilst there has been commendable progress in integrating TM into the legislative framework by introducing the Traditional Health Practitioners Act 22 of 2007, there has been slow progress in implementing

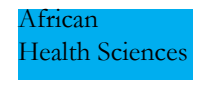

3100
(C) 2019 Nompumelelo et al. Licensee African Health Sciences. This is an Open Access article distributed under the terms of the Creative commons Attribution License (https://creativecommons.org/licenses/BY/4.0), which permits unrestricted use, distribution, and reproduction in any medium, provided the original work is properly cited. 
this Act. The purpose of the Act is to create an Interim Traditional Health Practitioners Council responsible for providing a regulatory framework to ensure the management and control of registration, training and conduct of practitioners, students and special categories in the THP profession. $^{4}$

Recently, resulting from the inauguration of the Interim Council in 2013 which came into effect on 1 May 2014, there has been an accelerated enthusiasm for the implementation of the Act. ${ }^{5,6,7}$ However, little change has been noted on the ground due to the lack of empirical data on TM systems to inform implementation ${ }^{8}$ and to the lack of substantive details on the applicability of the proposed regulations. ${ }^{5}$ One factor that affects the registration and training requirements stipulated by the THP Act is the articulation of core competencies to inform the training, accreditation and registration of THPs. Competencies can be defined as a combination of attributes such as knowledge, abilities, skills and attitudes which enable an individual to perform a set of tasks to an appropriate standard. ${ }^{8,9}$ Core competencies set clear guidelines for the skills needed to plan, implement and evaluate a training program and allow for precise decision making on professional development and training needs and for shaping training programs and qualifications. ${ }^{10}$

Defining core competencies is essential for the development of training curricula, for determining appropriate learning approaches, for creating assessment strategies and for institutional and professional development of the profession. This pedagogical approach to training is a norm for other conventional practices of health care. The Accreditation Council for Graduate Medical Education (ACGME), American Board for Medical Specialists (ABMS) and Healthcare Leadership Alliance (HLA) in the United States of America identify patient care, medical knowledge, practice-based learning, interpersonal and communication skills, professionalism, systems-based practice and business skills as the general competencies common in all physicians' training. ${ }^{11,12,13,14}$

The Medical and Dental Professions Boards of the Health Professions Council of South Africa identified core competencies for undergraduate training in nursing, dentistry and medicine as:

1. Giving optimal, ethical, comprehensive and patient/ client-centred care in a plurality of health and social contexts;

2. Acquiring the skills, knowledge, attitude and character appropriate to their practice;
3. Performing comprehensive assessments of patients/ clients;

4. Using preventative, promotive, therapeutic and rehabilitative interventions effectively;

5. Demonstrating appropriated use of procedural skills, both diagnostic and therapeutic;

6. Developing rapport, trust and ethical therapeutic relationships with patients, families and communities;

7. Accurately eliciting and synthesising relevant information on and the perspectives of patients, families and communities;

8. Conveying relevant information and explanations accurately and effectively to patients and families;

9. Responding to individual patient health needs and related issues as part of holistic care ;

10. Demonstrating commitment and accountability to patients through ethical practice.

In South Africa, there is limited documentation of THP competencies. This study focused on the identification of existing core competencies that are necessary for the apprentice THPs to be deemed competent to practice.

\section{Methods}

This study investigated the dynamics of the indigenous training of THPs in rural, peri-urban and urban areas of EThekwini Municipality and UThungulu District, KwaZulu-Natal Province, South Africa.

Firstly, purposeful sampling was used to identify THP tutors (Ogobela in Zulu language) with THP training sites and Amathwasa (initiates) undergoing training. The process included networking by contacting known THPs in the communities, attending Imigido (traditional healer ceremonies) and THPs associations' meetings in order to request volunteers for the study. There is no known formal Ogobela register; hence the study did not pre-determine the study sample size. Initially 10 THP tutors volunteered and enrolled for the study. The second phase of recruitment was convenient sampling in which Ogobela gave consent for the recruitment of Amathwasa under their apprenticeship. The third phase used snowball sampling in which the sampled participants recommended further participants for the study.

The data collection took place between March 2017 and June 2017 using a mixed method approach. Initially, a semi-structured questionnaire with close-ended and open-ended questions was administered to the THPs and their trainees. Subsequently, an exploratory descrip- 
tive qualitative research was undertaken. This phase utilised guided journaling where the Ogobela and Amathwasa were provided with journaling booklets comprising topic guides in which they could record their daily activities. Amathwasa completed at least three journaling booklets over a period of three months resulting in an average of sixty days of journaling per participant. Ogobela were required to complete one journaling booklet for the duration of the study.

The Traditional Health Practitioners Association Executive Committee of eThekwini and the University of KwaZulu-Natal Biomedical Research Ethics committee (BREC) gave permission to for the study.

\section{Results}

Of the 74 participants, $36(49 \%)$ were Ogobela and 38 (51\%) were initiates (Amathwasa). Nearly two-thirds (57\%) of the participants were from urban central and peri-urban areas whilst $43 \%$ were from rural areas. The majority $(86 \%)$ were females. Of these initiates, $28 \%$ were in level 1 of initiation, termed "isigaba sebomvu," in which initiates wear red clothing and red clay (ibomvu). Thirty one percent were in level 2, termed "isigaba somcako," in which white clothing and white clay (umcako) is worn and 18\% were in level 3, termed "isigaba sokuphothula or sentwaso," in which the initiate is made ready for the initiation ceremony. Twenty three percent $(23 \%)$ were in level 4 , which is the stage immediately after the initiation ceremony. In this level, an initiate is operational under the apprenticeship of the trainer. Initiation periods ranged from 0-3 months (13\%), 3-5 months (21\%), $2-3$ years $(18 \%)$, and the majority were initiated for more than 3 years $(33 \%)$.

\section{Ogobela initiation and experience}

The level of training and initiating experience of Ogobela ranged from $0-10$ years $(38 \%)$ to $10-20$ years $(62 \%)$. At least $38 \%$ of Ogobela had one initiate under apprenticeship, $17 \%$ had two, 20\% had four and the remaining 25\% had up to eight initiates at the time of the study.

Nearly all (97\%) Ogobela were initiated through the process of apprenticeship whilst the remaining 3\% reported that they were guided by the ancestors in acquiring the knowledge and skill necessary to practice. The pathway of becoming a trainer (Ogobela) was not an exclusive process hence all participating trainers were included in the study. However, a significant divergence of data was identified between the two groups. Those initiated through apprenticeship strived to strike a balance between the comprehensive acquisition of skills and competencies on the one hand, and appeasing and invoking the ancestors on the other. The group that was initiated through ancestral guidance focused more on appeasing and invoking the ancestors to guide the initiation and training process. The latter group believed that the ancestors are supposed to teach all the necessary skills for one to be competent. Nonetheless, convergence was identified between both groups in relation to the expected core competencies an individual should master to become a competent and qualified healer.

\section{Traditional Health Practitioner Core Competencies}

The following eleven core competencies and their enabling skills for THPs were identified.

\section{i. Consultation}

According to Ogobela, consultation called ukuxhumana nesiguli/ukubonana nokuxoxa nesiguli) is the first step in the healing process. This information was validated by the convergence of data from the journaling booklets in which Ogobela documented consultation as their initial teaching and Amathwasa documenting it as the first lesson of their learning process. Predominantly taking place in the consultation room of the healer known as "isigodlo," this process is significant for gaining patient rapport and for determining the patient's genealogy, behavioural habits, lifestyle and social environment.

The following skills were identified as essential:

a. provision of empathetic and patient-centred discussions;

b. demonstration of open, unbiased and honest communication;

c. demonstration of non-judgmental behaviour; d. identifying and observing ethical dilemmas in patient relations and care and

e. observing confidentiality (ukuba nesifuba).

\section{ii. Diagnoses (ukublola/ukublabluba/ukubhula)}

In this process, an ithwasa is taught to use divination through oracles to foretell patients' problems and diagnose illnesses. Enabling skills included the ability to a. channel ancestral communication, which is a process of directing cosmic energies normally induced by burning incense (impepho),

b. recognise signs of ancestral communication through oracles and nature, 
c. identify and distinguish illnesses and their causal factors and

d. observe discretion and confidentiality (ukuba nemfiblo).

\section{iii. Holistic patient care and treatment}

Holistic patient care and treatment is called ukunakekela isiguli. According to the participants, this includes ensuring complete wellbeing of patients. It is based on principles of wholeness that take into cognisance patients' cultural, physical, emotional and psychological wellness. The initiate is required to

a.identify genealogical factors;

b.understand illnesses and contributing cultural factors;

c.have knowledge of culture and the relevant ritualistic requirements;

d.provide appropriate guidance in relation to healing;

e.identify and align appropriate treatment for specific illnesses and

f.provide culturally relevant palliative care.

\section{iv. Integrative healing}

Integrative healing covers historical, genealogical and interpersonal factors that affect the individuals' physical, psychological and emotional wellbeing. Hence, the family is also included in the healing process. This requires the initiate to

a. identify and integrate psychological, spiritual and genealogical determinants into healing;

b. understand and apply knowledge of natural elements such as water, earth, air and light into healing;

c. incorporate healing within the context of integrated spiritual development;

d. integrate family into the healing process;

e. conduct family counselling;

f. observe and understand the cultural determinants of illnesses and

g. provide treatment counselling.

\section{v. Application of healing procedures, methods and cultural rituals}

According to Ogobela the initiate is supposed to have knowledge of different healing methods and an understanding of relevant cultural rituals. Each initiate is supposed to be competent in the application of acquired knowledge to the healing procedures and cultural rituals used. This includes; a. application of appropriate therapies for the relevant illnesses;

b. use and application of different cultural rituals to different circumstances;

c. understanding the significance of cultural rituals for diverse healing scenarios;

d. understanding appropriate therapeutic interventions;

e. knowledge of prophylactic, preventative and rehabilitative interventions (ukuqinisa, ukuvikela nokulapha);

f. understanding patient care including the follow-ups and continuity of procedures;

g. distinguishing between conventional and culture bound illnesses and

h. understanding restorative interventions and cleansing (ukugeza, ukublambulula).

\section{vi. Spiritual development (ukukbula ngokomoya)}

Promotion of spiritual development for the individual patient, family and community was identified as a key competency. The following enabling skills are required:

a. ability to provide spiritual counselling;

b. knowledge and understanding of cultural determinants that pose a threat to spiritual development and wellbeing; c. understanding uncertainties and sensitivities related to spirituality and culture, and

d. observing respect for cultural differences and family rituals.

\section{vii. Ethical practice and professionalism (ukuriphatha)} Initiates are required to apply the ethical principles they are taught during the process of initiation. This requires the THP to:

a. promote Ubuntu in their practice;

b. facilitate communication with patients and among families and communities;

c. promote a good rapport;

d. observe honesty in all dealings;

e. hold respect for other healers, patients and ancestors and

f. develop positive relations with patients and their families based on honesty, empathy, trust and respect.

\section{viii. Problem solving}

Proficiency in problem solving and decision-making emerged as a core competency for Traditional Health practice. The following skills make up this core competency: 
a. facilitation of family rehabilitation, reconciliation and rectification of ancestral indiscretions (inblambuluko);

b. facilitation and promotion of cultural restoration (ukwenza imisebenzi imiseben₹i);

c. promoting and influencing behavioural changes and

d.facilitation of family and couples counselling.

\section{ix. Knowledge of herbs, traditional cures and appli- cable therapies.}

According to the participants, this process, known as ukuthaka imithi, involves the use of plant material to make healing extracts and mixtures. The enabling skills considered essential include the ability to:

a. identify and distinguish different herbs, plants and trees (ukublukanisa imithi);

b. master and apply muthi harvesting techniques (Ukumbiwa nokukbiwa kwemithi);

c. prepare and process different muthi;

d. mix and combine different combinations of different herbs (Ukuthaka imithr) and

e. apply and use different herbal combinations for a variety of illnesses and problems.

\section{x. Ancestral philosophy and procedures}

A competent THP is required to have comprehensive knowledge of ancestral procedures and rituals. The following ancillary skills were reported as key to achieving the desired core competency:

a. understanding types and categories of ancestral spirits (imimoya);

b. preparation and use of izinkamba which is a combination of plants specifically used for invoking ancestors; c. types of plants used (impande);

d.knowledge and understanding of ancestral rituals;

e. spiritual communication and channelling (ukuxbumana ne Dlo:i) and

f. knowing the use and significance of animals in ancestral rituals.

\section{xi. Preventative and protective methods and proce- dures (ukuqiniswa nokuvikelwa kweriguli)}

Knowledge of preventative and protective methods was also identified as an essential skill that Amathwasa were required to master during their training. The ancillary competencies required included:

a. the ability to provide counselling on behavioural change and coping mechanisms for patients; b. knowledge of prophylactic methods applicable to different culture-bound illnesses and conditions;

c. knowledge of applicable treatment rituals for culture-bound illnesses and

d. knowledge of different methods of prevention.

\section{Discussion}

The persistence of African TM through centuries of subjugation by allopathic medicine implies that there are fundamental pillars sustaining the system. Traditional Medicine is commonly associated with herbalism, ancestral spiritual components and culture, but it is also seen as neglecting certain factors contributing towards the sustainability of the system particularly those linked to training and initiation. This study identified apprenticeship as being a well-defined component of TM that contributes to its tenacity by ensuring the competency of its THPs. In contrast to conventional health practice, which has at least ten core competencies, this study identified eleven core competencies within the TM context. The identified competencies are consultation, diagnoses, holistic patient care and treatment, integrative and holistic healing, application of healing procedures, methods and cultural rituals, spiritual development, ethical and professional competencies, problem solving, herbalism, ancestral knowledge and end of life care. These competencies have identifiable commonalities with conventional competencies such as diagnosis, patient care, medical knowledge, practice-based learning and improvement, interpersonal and communication skills, and professionalism. ${ }^{9,10,11}$. However, some differences in competency requirements were identified between the two health care systems. The TM competencies include a comprehensive, integrative and spiritual component that incorporates family into the healing process, whilst the conventional competencies are less comprehensive and more individualistic.

The introduction of the South African Traditional Health Practitioners Act 22 of 2007 was aimed regulating registration, training and conduct of TM practitioners and students and providing guidelines for the accreditation of THPs. To date there the regulatory process is yet to be implemented; there has been no progress on the accreditation of the THPs and there is no predetermined content for accreditation. Falling short of a predetermined context, the Council is unable to effectively regulate THPs and accredit TM training institutions. The 
paucity of empirical information on the training competencies contributes largely to the lack of guidelines for curriculum accreditation. Indigenous African Traditional Medicine training core competency requirements and capacitation is based on recognised training approaches and methods. These are not unique to TM system as they are also recognisable within the conventional systems used within the business and health sectors in a form of mentoring, coaching and practical. The identified training core competencies form a foundation for a framework of an accredited training programme and can be effectively used to further facilitate implementation of THP accreditation programs.

\section{Conclusion}

Traditional Medicine training is based on fundamental pillars as identified in the training competencies. Its core competencies are not unique to THP practice as they are also identifiable in conventional health practice. If the Traditional Health Practitioners Act 22 of 2007 is to achieve its purpose of providing for the management of and control over the registration, training and conduct of practitioners, it would be appropriate for the appointed Interim THP Council to include the identified competencies in its criteria for the accreditation of training and assessments.

\section{Conflict of interest/competing interests}

The authors declare that they have no conflict of interest

\section{References}

1. Gqaleni N, Moodley I, Kruger H, Ntuli A, McLeod H. Traditional and complementary medicine: health care delivery. In: Harrison S, Bhana R, Ntuli A, editors. Durban; South African Health Review. 2007:175-188.

2. World Health Organization. The Promotion and Development of Traditional Medicine: A report of WHO meeting Geneva; 1978.

3. World Health Organization. Legal Status of Traditional Medicine and Complementary/Alternative Medicine: A Worldwide Review Geneva; 2001. http://apps.who. int/medicinedocs/pdf/h2943e/h2943e.pdf. (accessed 18 December 2017)

4. South African. Department of Health. Traditional Health Practitioners Act No. 22 of 2007.

5. Street, RA. Unpacking the new proposed regulations for South African Traditional Health Practitioners. South African Medicine Journal. 2016; 106 (4): 325-326.

6. South African Department of Health. Interim Traditional Council Ianugurated.2013. Http://www.sabinetlaw.co.za/health/articles/interim-health-coucil-inaugurated (accessed February 2017)

7. President of the Republic of South Africa, Commencement of certain sections of the Traditional Health Practitioners Act Proclamation, 2007 Notice No.29 of 2014. In: Government Gazette No.37600.Pretoria: Government Printing Works. 2014

8. Shilton, T., Howat, P., James, R. and Lower, T. Health promotion development and health promotion workforce competency in Australia. Health Promotion Journal of Australia. 2001; 12(2): 16-123.

9. Farley M, Clewley G. Project to undertake research relating to core public health competencies. Project Report for the Ministry of Health.2005: 59. Cited in Generic Competencies for Public Health in Aotearoa-New Zealand. Public Health Association of New Zealand. 2007. http://www.pha.org.nz/documents/GenericCompetencies forPublicHealthMarch2007.pdf

10. Dempsey, Colette; Barry, Margaret M.; Battel-Kirk, Barbara. Literature Review: Developing Competencies for Health Promotion. International Union for Health Promotion and Education and the Health Promotion Research Centre. 2010:1-132.https://aran.library.nuigalway. ie/.../4442/CompHP_Literature_Review_Part_1.pdf?

11. Nompumelelo Mbatha, Renée A Street, Mlungisi Ngcobo, Nceba Gqaleni. Sick certificates issued by South African Traditional Health Practitioners: Current legislation, challenges and the way forward. S Afr Med J. 2012; 102:129-131 PubMed .

12. Health Professions Council of South Africa. Core competencies for undergraduate students in clinical associate, dentistry and medical teaching and learning programmes in South Africa. Medical and dental professions Boards of the health Professions Council of South Africa. 2014:1-14. http://www.hpcsa.co.za/uploads/editor/UserFiles/downloads/medical_dental/MDB $\% 20$ Core $\% 20$ Competencies $\% 20-\% 20$ ENGLISH $\% 20-\% 20$ FINAL\%202014.pdf ( accessed December 2017) 13. Lurie, S.J. et al. Measurements of the General Competencies of the Accreditation council for Graduate Medical Education: A Systematic Review. Academic Medicine. 2009; 84, No3: 301-309. https://www.ncbi.nlm.nih.gov/ 
pubmed/19240434. (accessed December 2017)

14. Combes, J.R. and Arespacochaga, E. Physicians competencies for a 21st Century health Care System. Journal of Graduate Medical Education. 2012:401-403. https://
www.ncbi.nlm.nih.gov/pmc/articles/PMC3444207/ (accessed February 2017).

15. Stefl, E.M. Common Competencies for All Healthcare Managers: The healthcare leadership Alliance Model. Journal of Healthcare Management. 2008; 53(6):360-374. 